Old Dominion University

ODU Digital Commons

\title{
Extracting the Number of Short Range Correlated Nucleon Pairs from Inclusive Electron Scattering Data
}

R. Weiss

A. W. Denniston

J. R. Pybus

O. Hen

E. Piasetzky

See next page for additional authors

Follow this and additional works at: https://digitalcommons.odu.edu/physics_fac_pubs

Part of the Elementary Particles and Fields and String Theory Commons, Nuclear Commons, and the Quantum Physics Commons 


\section{Authors}

R. Weiss, A. W. Denniston, J. R. Pybus, O. Hen, E. Piasetzky, A. Schmidt, L. B. Weinstein, and N. Barnea 


\title{
Extracting the number of short-range correlated nucleon pairs from inclusive electron scattering data
}

\author{
R. Weiss, ${ }^{1}$ A. W. Denniston, ${ }^{2}$ J. R. Pybus $\odot,{ }^{2}$ O. Hen $\odot,{ }^{2}$ E. Piasetzky, ${ }^{3}$ A. Schmidt $\odot,{ }^{4}$ L. B. Weinstein $\odot,{ }^{5}$ and N. Barnea ${ }^{1, *}$ \\ ${ }^{1}$ The Racah Institute of Physics, The Hebrew University, Jerusalem 9190401, Israel \\ ${ }^{2}$ Massachusetts Institute of Technology, Cambridge, Massachusetts 02139, USA \\ ${ }^{3}$ School of Physics and Astronomy, Tel Aviv University, Tel Aviv 69978, Israel \\ ${ }^{4}$ George Washington University, Washington, DC 20052, USA \\ ${ }^{5}$ Old Dominion University, Norfolk, Virginia 23529, USA
}

(Received 19 May 2020; revised 12 January 2021; accepted 23 February 2021; published 15 March 2021)

\begin{abstract}
The extraction of the relative abundances of short-range correlated (SRC) nucleon pairs from inclusive electron scattering is studied using the generalized contact formalism (GCF) with several nuclear interaction models. GCF calculations can reproduce the observed scaling of the cross-section ratios for nuclei relative to deuterium at high $x_{B}$ and large $Q^{2}, a_{2}=\left(\sigma_{A} / A\right) /\left(\sigma_{d} / 2\right)$. In the nonrelativistic instant-form formulation, the calculation is very sensitive to the model parameters and only reproduces the data using parameters that are inconsistent with $a b$ initio many-body calculations. Using a light-cone GCF formulation significantly decreases this sensitivity and improves the agreement with ab initio calculations. The ratio of similar mass isotopes, such as ${ }^{40} \mathrm{Ca}$ and ${ }^{48} \mathrm{Ca}$, should be sensitive to the nuclear asymmetry dependence of SRCs, but is found to also be sensitive to low-energy nuclear structure. Thus the empirical association of SRC pair abundances with the measured $a_{2}$ values is only accurate to about $20 \%$. Improving this will require cross-section calculations that reproduce the data while properly accounting for both nuclear structure and relativistic effects.
\end{abstract}

DOI: 10.1103/PhysRevC.103.L031301

To a good approximation, neutrons and protons with momentum below the Fermi sea can be considered as independently moving in well-defined quantum orbits of the average, mean-field, nuclear interaction. Above the Fermi sea, short-range correlated (SRC) pairs dominate [1-10]. Therefore, quantifying the number of correlated pairs is important for obtaining a complete picture of the atomic nucleus.

A description of correlations in complex nuclear systems can be done in the spirit of the successful atomic theory, in which various properties of a unitary gas are connected to a single parameter, the contact [11-14]. In essence, the contact counts the number of SRC pairs in the system. The importance of this quantity to nuclear systems was demonstrated by the success of the generalized contact formalism (GCF), which takes into account the complicated nature of the nuclear force [8,15-20]. SRC pair abundances are also used in modeling the effective impact of SRCs on the nuclear symmetry energy and neutron-star properties [21-24], and in studies of the modification of quark distributions in nuclei [1,25-29], the flavor dependence of the European Muon Collaboration (EMC) effect [30-32], and low-energy QCD symmetry breaking mechanisms $[33,34]$.

Inclusive electron scattering $\left(e, e^{\prime}\right)$ measurements are commonly used to estimate SRC pair abundances in nuclei. In kinematics sensitive to SRCs, the cross-section ratio

*Corresponding author: nir@phys.huji.ac.il
$\sigma_{A} / A / \sigma_{d} / 2$, between nucleus $A$ and the deuterium, "scales," reaching a constant value independent of the momentum and energy transfer [30,35-39]. The value of this constant, $a_{2}(A / d)$ or simply $a_{2}$, is traditionally interpreted as the number of neutron-proton $(n p)$ deuteron-like SRC pairs in nucleus $A$ relative to deuterium [1,30,35-39].

This scaling is seen at a kinematic of $Q^{2} \gtrsim 1.4 \mathrm{GeV}^{2}$ and $1.5 \leqslant x_{B} \leqslant 1.9$, where $x_{B}=Q^{2} / 2 m \omega ; Q^{2}=\boldsymbol{q}^{2}-\omega^{2} ; \boldsymbol{q}$ and $\omega$ are the three-momentum and energy transfer, respectively; and $m$ is the nucleon mass. The value of $x_{B} \geqslant 1.5$ determines that the minimum allowed initial momentum $k_{\min }$ of the struck nucleon is very close to the typical nuclear Fermi momentum for medium to heavy nuclei, $k_{F} \approx 250 \mathrm{MeV} / \mathrm{c}$ [36]. Nucleons with higher momenta are predominantly part of deuteronlike SRC pairs [3-10]. The scaling then naturally arises in a simplistic SRC picture where the struck nucleon belongs to a stationary deuteronlike pair. In this picture the recoil momentum is carried by a single nucleon and the $A-2$ residual nucleus does not recoil. Therefore, $k_{\min }$ of the struck nucleon and its ground-state momentum distribution are similar in deuterium and heavier nuclei, resulting in cross-section ratio scaling that should be proportional to the number of SRC pairs $[35,36]$.

However, this intuitive interpretation of $a_{2}$ in terms of SRC abundances neglects important effects: (1) the presence of non-deuteron-like SRCs [proton-proton $(p p)$, neutronneutron $(n n)$, and $p n$ pairs with $s \neq 1]$; (2) pair center-of-mass (c.m.) motion [40]; and (3) possible excitation of the residual $A-2$ system. C.m. motion and $A-2$ excitation can 


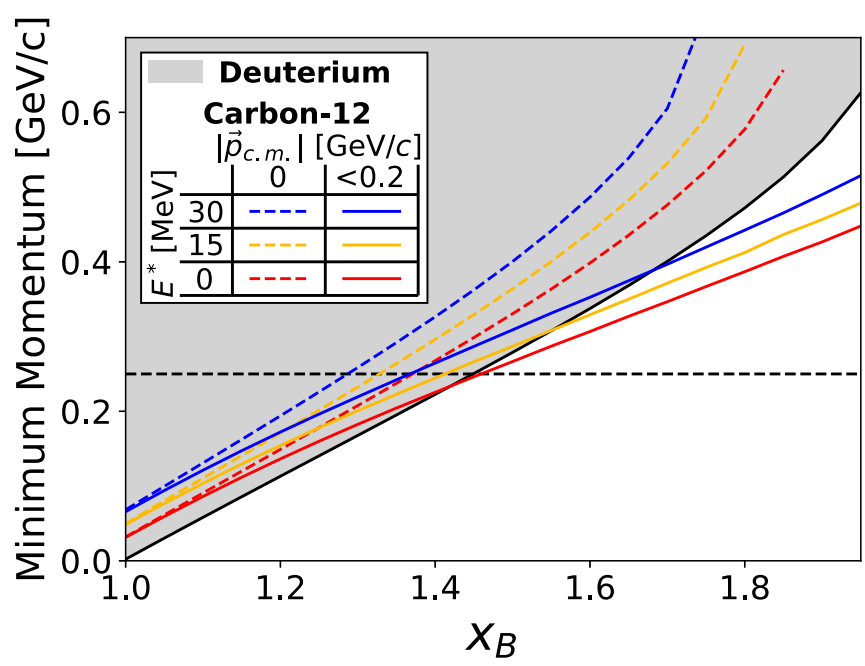

FIG. 1. The minimum possible momentum of the nucleon absorbing the virtual photon, $k_{\min }$, in inclusive scattering as a function of $x_{B}$, for $Q^{2}=2 \mathrm{GeV}^{2}$. The black line shows $k_{\min }$ for the deuteron, while the colored lines show $k_{\min }$ for SRC pairs in ${ }^{12} \mathrm{C}$, for different $A-2$ excitation energies, $E_{A-2}^{*}$, and for different pair center-of-mass momenta, denoted by $\left|\vec{p}_{\text {c.m. }}\right|$. The gray region shows the initial momentum range, $k \geqslant k_{\min }$, for $d\left(e, e^{\prime}\right)$. The horizontal dashed line corresponds to the Fermi momentum for heavy nuclei, $k_{F} \approx 0.25 \mathrm{GeV} / \mathrm{c}$.

dramatically affect $k_{\min }$ (see Fig. 1) which can significantly affect the simplistic interpretation of $a_{2}$.

In addition, final-state interaction (FSI) can contribute to the measured $\left(e, e^{\prime}\right)$ cross sections and disrupt this simplistic interpretation of $a_{2}$. While such contributions grow with $x_{B}$ and can reach up to $50 \%$, it was argued by several calculations [35,41-46] (but not all [45]) that they are confined to within SRC pairs and cancel to first approximation in the $A / d$ ratio. The main inputs for the FSI calculations are measured $N N$ scattering cross sections and these calculations are done in a high-resolution reaction model using one-body reaction operators, similar to the reaction scheme employed by our GCF calculations.

As more and better $a_{2}$ data are becoming available [47], and as studies utilizing $a_{2}$ values as SRC abundances demand higher precision [39], it is timely to examine the quantitative impact of realistic SRC modeling on the classical interpretation of $a_{2}$. Such modeling is also important for establishing a direct connection between inclusive electron scattering and $a b$ initio many-body structure calculations [5,18,48-53].

Here we study the interpretation of $a_{2}$ scaling using the GCF to calculate high- $x_{B}$ high- $Q^{2}$ inclusive scattering cross sections. By comparing measured and GCF-calculated cross sections using different model parameters we provide a new, quantitative, understanding of the model dependence of SRC pair abundance extraction.

The GCF is a realistic effective model of SRCs, used to connect experimental data and $a b$ initio nuclear structure calculations $[8,16,18]$. Building on the scale separation of nucleons in SRC pairs from the surrounding nuclear environment, it models nucleons in SRC pairs using universal (i.e., nucleus independent) two-particle functions, and systemand state-dependent contact terms that describe the abundance of SRC pairs. This scale-separated approach successfully reproduced $a b$ initio calculated nucleon distributions at short distance and high momentum, enabling a meaningful extraction of nuclear contact terms $[8,16,18]$. More recently, it was extended to model nuclear spectral and correlation functions $[17,19]$, enabling a successful reproduction of a wide range of $\left(e, e^{\prime} N\right)$ and $\left(e, e^{\prime} N N\right)$ measurements [8,9,15,19,20,54]. The GCF thus provides an established and robust formalism to describe experimental data using effective parameters obtained from many-body calculations.

To quantify the impact of these effects we perform GCF calculations of inclusive cross-section ratios using various parameters and compare them to each other and to experimental data. We used both nonrelativistic instant-form (IF) and lightcone (LC) GCF formulations, to see the effect of relativistic corrections for these high-momentum nucleons. We integrated the previously derived GCF $\left(e, e^{\prime} N\right)$ and $\left(e, e^{\prime} N N\right)$ cross sections over the knocked-out nucleons, to obtain the inclusive $\left(e, e^{\prime}\right)$ cross section.

Within the plane-wave impulse approximation (PWIA), the IF GCF $\left(e, e^{\prime} N N\right)$ cross section for the breakup of an SRC pair is given by [54]

$$
\begin{aligned}
& \frac{d^{8} \sigma_{A}}{d E_{e} d \Omega_{e} d^{3} \vec{p}_{\mathrm{c} . \mathrm{m} .} d \Omega_{\mathrm{rel}}} \\
& =\kappa_{\mathrm{IF}} \sum_{N_{1} N_{2}, \beta} s \sigma_{e N_{1}} C_{N_{1} N_{2}}^{A, \beta}\left|\tilde{\varphi}_{N_{1} N_{2}}^{\beta}\left(\vec{p}_{\mathrm{rel}}\right)\right|^{2} n_{N_{1} N_{2}}^{A, \beta}\left(\vec{p}_{\mathrm{c} . \mathrm{m} .}\right) \\
& \equiv \sum_{N_{1} N_{2}, \beta} C_{N_{1} N_{2}}^{A, \beta} \times \sigma_{N_{1} N_{2}, \mathrm{IF}}^{\beta},
\end{aligned}
$$

where $E_{e}$ and $\Omega_{e}$ are the energy and solid angle of the scattered electron, and $\vec{p}_{\text {c.m. }}$ and $\vec{p}_{\text {rel }}$ are the c.m. and relative momenta of the initial-state SRC pair, respectively. $\sigma_{e N_{1}}$ is the off-shell electron-nucleon cross section, $s$ is a symmetry factor $\left(s=1\right.$ for $n p$ and $p n$ and $s=2$ for $n n$ and $p p$ ), and $\kappa_{\mathrm{IF}} \equiv$ $\frac{1}{32 \pi^{4}} \frac{p_{\text {rel }}^{3} E_{1}^{\prime} E_{2}}{\left|\left(E_{2} \vec{p}_{1}^{\prime}+E_{1}^{\prime} \vec{p}_{2}\right) \cdot \vec{p}_{\text {rel }}\right|}$ is a phase-space factor, where $\left(\vec{p}_{1}^{\prime}, E_{1}^{\prime}\right)$ and $\left(\vec{p}_{2}, E_{2}\right)$ are the knocked-out and spectator nucleon fourmomenta, respectively. $\left|p_{\text {rel }}\right|$ is fixed by energy-momentum conservation.

$C_{N_{1} N_{2}}^{A, \beta}$ are nucleus-dependent nuclear contacts, measuring the probability to find an $N_{1} N_{2}$ SRC pair ( $p p, n n, n p$, or $p n$ ) in nucleus $A$ with quantum numbers $\beta . \beta=1$ denotes spin1 deuteronlike pairs, and $\beta=0$ is for the spin-zero $s$-wave pairs. $n_{N_{1} N_{2}}^{A, \beta}\left(\vec{p}_{\text {c.m. }}\right)$ is the SRC pairs c.m. momentum distribution, approximated by a three-dimensional Gaussian with an $A$-dependent width $\sigma_{\text {c.m. }}[40,44,55] . \tilde{\varphi}_{N_{1} N_{2}}^{\beta}$ are the universal two-body functions of the relative momentum distribution of nucleons in SRC pairs, obtained by solving the zero-energy two-body Schrödinger equation with a given $N N$ interaction model (e.g., AV18, N2LO, etc.).

We stress that the contact values are fixed by comparison with $a b$ initio calculations [18] and $\sigma_{\text {c.m. }}$. was measured in Ref. [40]. The unmeasured average excitation energy of the residual system $E_{A-2}^{*}$ is limited by the typical excitation energy of the system $\left(0 \leqslant E_{A-2}^{*} \leqslant 30 \mathrm{MeV}\right)$. The uncertainties 
of these parameters are used to evaluate the uncertainties of the GCF calculations.

Light-cone four-momentum vectors are expressed in terms of longitudinal (along the $\boldsymbol{q}$ direction) plus- and minusmomentum $p^{ \pm} \equiv p^{0} \pm p^{3}$ and transverse momentum $\vec{p}^{\perp} \equiv$ $\left(p^{1}, p^{2}\right)$. The light-cone momentum fraction is $\alpha \equiv p^{-} / \bar{m}$, where $\bar{m}=m_{A} / A$. The advantages of studying inclusive reactions using LC are discussed in Ref. [35]. [54]

The PWIA LC GCF $\left(e, e^{\prime} N N\right)$ cross section is given by

$$
\frac{d^{8} \sigma_{A}}{d E_{e} d \Omega_{e} d^{3} \vec{p}_{\mathrm{c} . \mathrm{m} .} d \Omega_{\mathrm{rel}}}=\sum_{\beta} C_{N_{1} N_{2}}^{A, \beta} \times \sigma_{N_{1} N_{2}, \mathrm{LC}}^{\beta},
$$

where

$$
\sigma_{N_{1} N_{2}, \mathrm{LC}}^{\beta}=s \kappa_{\mathrm{LC}} \sigma_{e N_{1}} \psi_{N_{1} N_{2}}^{\beta}\left(\alpha_{\mathrm{rel}}, \vec{p}_{\text {rel }}^{\perp}\right) \rho_{N_{1} N_{2}}^{A, \beta}\left(\alpha_{\text {c.m. }}, \vec{p}_{\mathrm{c} . \mathrm{m} .}^{\perp}\right) .
$$

Here $\alpha_{\text {c.m. }}, \vec{p}_{\text {c.m. }}^{\perp}, \alpha_{\text {rel }}$, and $\vec{p}_{\text {rel }}^{\perp}$ are the LC longitudinal, LC transverse, c.m., and relative momenta of the SRC pair, respectively. $\kappa_{\mathrm{LC}}=\kappa_{\mathrm{IF}} \frac{8 \pi^{3} \alpha_{A-2}}{\alpha_{1} \alpha_{\mathrm{c}, \mathrm{m}} E_{A-2}}$ is a phase-space factor. $\rho_{N_{1} N_{2}}^{A, \beta}\left(\alpha_{\text {c.m. }}, \vec{p}_{\text {c.m. }}\right)$ is a three-dimensional Gaussian of width $\sigma_{\text {c.m. }}$ and $\psi_{N_{1} N_{2}}^{\beta}\left(\alpha_{\text {rel }}, \vec{p}_{\text {rel }}^{\perp}\right)=\frac{\sqrt{m_{N}^{2}+k^{2}}}{2-\alpha_{\text {rel }}} \frac{\left|\tilde{\varphi}_{N_{1} N_{2}}^{\beta}(k)\right|^{2}}{(2 \pi)^{3}}$ is the LC equivalent of the IF universal function [3] where $k=\frac{m^{2}+k_{\perp}^{2}}{\alpha_{\mathrm{rel}}\left(2-\alpha_{\mathrm{rel}}\right)}-m^{2}$.

By integrating Eq. (1) or (2) we obtain the IF or LC GCF inclusive cross section:

$$
\frac{d^{3} \sigma_{A}}{d E_{k^{\prime}} d \Omega_{k^{\prime}}}=\sum_{N_{1} N_{2}, \beta} C_{N_{1} N_{2}}^{A, \beta} \int \sigma_{N_{1} N_{2}}^{\beta} d^{3} \vec{p}_{\mathrm{c} . \mathrm{m} .} d \Omega_{\mathrm{rel}},
$$

where the sum spans $s=1 n p$-SRC and $s=0 n p-, p p-$, and $n n$-SRC pairs and includes the electron coupling to either nucleon of the pair. The integration is limited by energymomentum conservation and depends on $\sigma_{\text {c.m. }}$ and $E_{A-2}^{*}$.

For the simple case of interacting with standing (i.e., no pair c.m. motion) on-shell (i.e., no $E_{A-2}^{*}$ effects) SRC pairs, the cross-section ratio for nucleus $A$ relative to deuterium is given by

$$
\frac{\sigma_{A}}{\sigma_{d}}=\frac{C_{p n}^{A, s=1}}{C_{p n}^{d, s=1}} \times\left[1+\frac{C_{p n}^{A, s=0}}{C_{p n}^{A, s=1}} \frac{\Psi_{p n}^{s=0}}{\Psi_{p n}^{s=1}}+2 \frac{C_{p p}^{A, s=0}}{C_{p n}^{A, s=1}} \frac{\Psi_{p p}^{s=0}}{\Psi_{p n}^{s=1}}\right],
$$

where the factor of 2 before the $p p$ term accounts for $n n$ pairs assuming isospin symmetry and $\Psi_{N_{1} N_{2}}^{\beta}$ represent the phasespace integral over the universal functions $\tilde{\varphi}_{N_{1} N_{2}}^{\beta}$ (instant form) or $\tilde{\psi}_{N_{1} N_{2}}^{\beta}$ (light cone). As $\frac{C_{N_{1} N_{2}}^{A, s=0}}{C_{p h}^{A, s=1}} \ll 1$ for any $N N$ interactions with a tensor force (for all $N_{1} N_{2}$ pairs), the cross-section ratio in this simplistic case will approximately equal $\frac{C_{p d}^{A, s=1}}{C_{p n}^{d s=1}}$. The latter was previously shown [18] to be insensitive to the $N N$ interaction model. It is thus expected for the $A / d$ cross-section ratio to be dominated by mean-field properties of the nucleus and thus be largely insensitive to the $N N$ interaction model $[18,28,53,57]$.

Figure 2 (top panels) shows the measured [38] and GCFcalculated $\sigma_{4} \mathrm{He} / 4 / \sigma_{D} / 2$ cross-section ratio, using nuclear contacts and c.m. width from Refs. [7,18,40], $E_{A-2}^{*}=0-30 \mathrm{MeV}$,

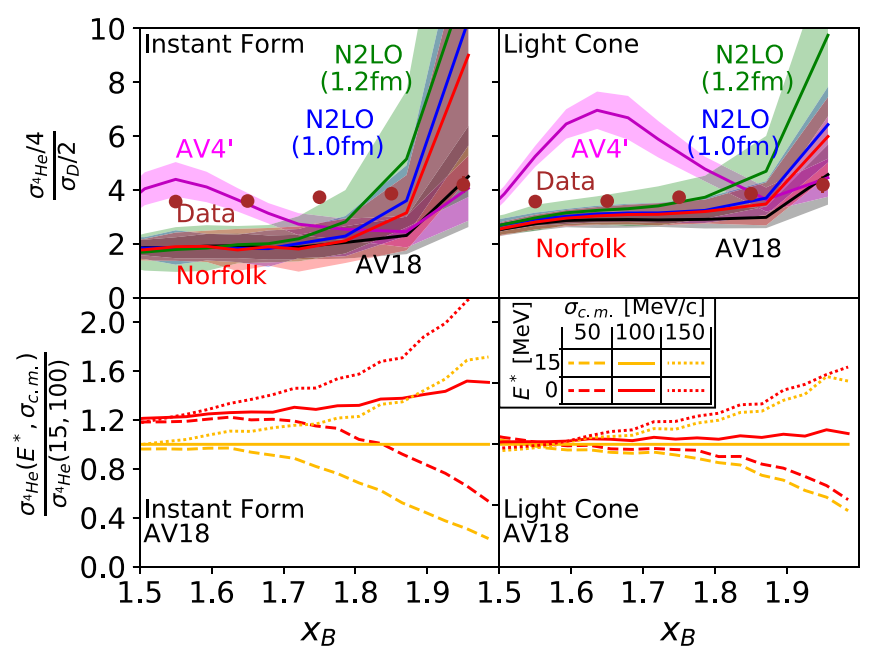

FIG. 2. Top: Measured per-nucleon $\left(e, e^{\prime}\right)$ cross-section ratios $\sigma_{4} \mathrm{He} / 4 / \sigma_{d} / 2$ as a function of $x_{B}$. The data [38] are compared with GCF calculations using both instant form (left) and light cone (right) GCF formulations with different $N N$ interaction models and using $\sigma_{\text {c.m. }}=100 \pm 20 \mathrm{MeV} / \mathrm{c}[7,40], E_{A-2}^{*}=0-30 \mathrm{MeV}$, and contact parameters from Ref. [18]. The widths of the bands show their $68 \%$ confidence interval due to the uncertainties in the model parameters. Bottom: Ratio of the GCF calculated ${ }^{4} \mathrm{He}$ cross section with different excitation energies $\left(E_{A-2}^{*}\right)$ and c.m. momentum distribution widths $\left(\sigma_{\mathrm{c} . \mathrm{m}}\right)$ to the cross section calculated for $E_{A-2}^{*}=15 \mathrm{MeV}$ and $\sigma_{\text {c.m. }}=100 \mathrm{MeV} / \mathrm{c}$. Calculations were done using both instant form (left) and light cone (right) GCF formulations with the AV18 [56] $N N$ interaction model.

and universal functions calculated with several $N N$ interaction models, including the phenomenological AV18 [56] and AV4' [58], and the chiral NV2 + 3-Ia* (Norfolk) [59-61] and N2LO [62-64] interaction with 1.0- and 1.2-fm cutoffs. Both IF and LC ratios show scaling plateaus (i.e., are constant for $1.4 \leqslant x_{B} \leqslant 1.9$ ), but the IF ratio is almost a factor of 2 too low. Calculations for additional nuclei are shown in the Supplemental Material [65].

The calculations are largely insensitive to the $N N$ interaction model, except for the special case of AV4' which does not include a tensor force and is therefore not dominated by deuteronlike pairs. This sensitivity of the GCF calculation to the tensor force stands in contrast with the effective field theory (EFT) analysis of Ref. [53] where the calculation does not directly employ high-resolution one-body reaction operators and the nature of the two-body interaction completely cancels in the cross-section ratio.

The marginal performance of the IF calculations is very surprising as they reproduce $\left(e, e^{\prime} N\right)$ and $\left(e, e^{\prime} N N\right)$ data at similar kinematics remarkably well [20,54]. The LC ratios are better, but are still $\approx 25 \%$ lower than the data. This might point to an issue with the contact extraction from ab initio calculations, because the results of Refs. [20,54] are not sensitive to the $A / d$ contact ratio. In the LC case, a 10-20\% relativistic correction to the contact extraction could explain the data.

To better understand this discrepancy we examined the impact of varying $\sigma_{\text {c.m. }}$ by $\pm 50 \mathrm{MeV} / \mathrm{c}$ and $E_{A-2}^{*}$ from 0 


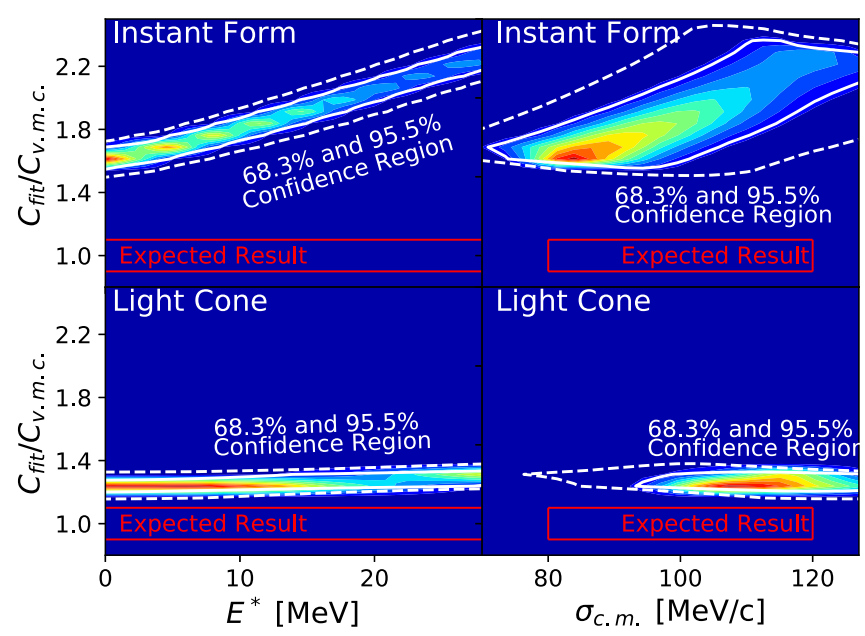

FIG. 3. GCF parameter confidence intervals for fitting ${ }^{4} \mathrm{He}\left(e, e^{\prime}\right) / d\left(e, e^{\prime}\right)$ data of Ref. [38] using instant form (top) and light cone (bottom) GCF formulations with the AV18 NN interaction [56]. The color scale represents the likelihood of the fit parameters given the data, with the white solid (dashed) contours indicating the $68.3 \%(95.5 \%)$ confidence regions. Red lines show the expected parameter values from previous measurements and/or $a b$ initio calculations [18]. The contact value $C_{n p}^{s=1}$ is shown as a ratio to its value extracted from many-body variational Monte Carlo (VMC) calculations. See text for details.

to $30 \mathrm{MeV}$ using the AV18 interaction [see Fig. 2 (bottom)]. The IF calculation is very sensitive to both parameters. A $15-\mathrm{MeV}$ change in $E_{A-2}^{*}$ changes the cross section by $\approx 20 \%$. A $50-\mathrm{MeV} / \mathrm{c}$ change in $\sigma_{\text {c.m. }}$. changes the cross section dramatically starting at $x_{B}=1.7$. Reference [66] also predicted large effects (up to $70 \%$ ) due to pair c.m. motion, which is very different than the $19 \pm 6 \% x_{B}$-independent correction used by Ref. [38], motivated by a simplistic onedimensional Gaussian smearing of the deuteron momentum distribution [67].

This sensitivity indicates that different effects, such as $A$ dependent FSIs [45], or contributions from $3 N$-SRCs that are missing in the current GCF calculations and are estimated to be $\mathrm{a} \approx 10 \%$ correction to the leading $2 N$-SRC contribution [8,10,37,68], might explain the disagreement seen in Fig. 2. The study of such corrections is ongoing and extends beyond the scope of the present paper. It also raises concerns about the ability to study the mass and asymmetry dependence of SRC pair abundances using pairs abundances extracted from $\left(e, e^{\prime}\right)$ measurements of light nuclei where $\sigma_{\mathrm{c} . \mathrm{m} .}$ and $E_{A-2}^{*}$ vary significantly.

Lastly we studied what parameter values are needed to describe the data. We varied $\sigma_{\mathrm{c} . \mathrm{m} .}, E_{A-2}^{*}$, and the spin-1 contact ratio $C_{n p}^{A, s=1} / C_{n p}^{d, s=1}$, to fit the ${ }^{4} \mathrm{He} / d[38]$ and ${ }^{12} \mathrm{C} / d$ data [30]. We kept the $C_{n p}^{s=1} / C_{N N}^{s=0}$ ratio fixed. The IF and LC results both described the data well [65].

The resulting ${ }^{4} \mathrm{He}$ AV18 parameters and their correlations are shown in Fig. 3. Results for the other $N N$ interaction models and different nuclei are shown in Table I of Ref. [65]. The fitted contacts have much larger uncertainties (up to $30 \%$ for IF and just under $10 \%$ for LC) than the typical $2 \%$ ex- perimental uncertainties in $a_{2}$. For the LC case this comes primarily from $\sigma_{\text {c.m. }}$, but IF is also sensitive to $E_{A-2}^{*}$.

The fitted IF contact ratios for deuteronlike $n p$ pairs are higher than the VMC calculation results by $50-150 \%$ for both $N N$ interactions and both nuclei, as expected from the results of Fig. 2. The fitted LC contacts are only 20-30\% higher than the VMC calculations for both $N N$ interactions, which is not much more than the $\approx 10 \%$ uncertainties on both the calculated and fitted contacts. For ${ }^{12} \mathrm{C}$ the same holds true for AV18 but a larger $80 \%$ disagreement is observed for N2LO.

Comparing with $a_{2}$, that is traditionally interpreted as a measure of deuteronlike $n p$ pairs, the fitted values are within $10-15 \%$ of the data for both ${ }^{4} \mathrm{He}$ and ${ }^{12} \mathrm{C}$, except for IF $\mathrm{N} 2 \mathrm{LO}$, which is within $\approx 30 \%$. However, this is an accidental result of the cancellation between the effects of $\sigma_{\text {c.m. }}$ and the contribution of non-deuteron-like pairs, which increase the ratio, and the effect of $E_{A-2}^{*}$, which decreases the ratio. This cancellation should be quite different in light and asymmetric nuclei where $\sigma_{\text {c.m. }}, E_{A-2}^{*}$, and the $n p / p p$-pair ratio can change rapidly with $A$.

To examine the effect of the nuclear asymmetry, we analyzed recent measurements of $a_{2}\left({ }^{48} \mathrm{Ca} /{ }^{40} \mathrm{Ca}\right)$ [39]. The calculation used ${ }^{40} \mathrm{Ca}$ contacts from Ref. [18] and assumed the same spin-zero contact for ${ }^{48} \mathrm{Ca}$. We varied the spin- ${ }^{48} \mathrm{Ca}$ contact and the values of $E_{A-2}^{*}$ and $\sigma_{\text {c.m. }}$ for each nucleus.

The calculation was relatively insensitive to $E_{A-2}^{*}$ and $\sigma_{\text {c.m. }}$. However, it could not place a stringent constraint on the important ${ }^{48} \mathrm{Ca} /{ }^{40} \mathrm{Ca}$ spin-1 contact ratio, because that is extremely sensitive to the parameter differences between ${ }^{48} \mathrm{Ca}$ and ${ }^{40} \mathrm{Ca}, \Delta \sigma_{\text {c.m. }}=\sigma_{\text {c.m. }}^{48 \mathrm{Ca}}-\sigma_{\text {c.m. }}^{40 \mathrm{Ca}}$, and $\Delta E^{*}=E_{46 \mathrm{~K}}^{*}-E_{38 \mathrm{~K}}^{*}$ (see Fig. 4). A 10-MeV change in either parameter difference induces a large change in the extracted contact ratio. This few$\mathrm{MeV}$ nuclear structure difference could plausibly be caused by the neutron skin of ${ }^{48} \mathrm{Ca}$ and the very different energy levels of ${ }^{38} \mathrm{~K}$ and ${ }^{46} \mathrm{~K}$.

This again emphasizes the large model dependence of interpretations of the measured nuclear asymmetry dependence of $a_{2}$, even in similar mass nuclei. This has direct implications for studies that use the asymmetry dependence of $a_{2}$, e.g., for understanding the flavor dependence of the EMC effect [31] and the properties of nucleons in dense neutron-rich matter $[21,23,24,69,70]$.

For completeness we note that the inclusive cross sections can also be analyzed in a complementary low-resolution picture with many-body operators and no SRCs [71]. This has not been implemented in the GCF and goes beyond the scope of the current paper. In addition, calculations in EFT approximate $a_{2}$ using the ratio of two-nucleon densities at short distance for nucleus $A$ and the deuteron [28,53]. This approach reproduces $a_{2}$ values, but cannot model the $x_{B}$ or $Q^{2}$ dependences of the ratio or provide insight into specific pair characteristics such as $\sigma_{\text {c.m. }}$ and the relation between $a_{2}$ values and low-energy nuclear structure (i.e., impact of $E_{A-2}^{*}$ ).

To conclude, $a_{2}$ measurements are widely used to extract SRC abundances, with wide ranging implications. Our calculations suggest that the traditional interpretation of $a_{2}$ as an empirical measure of the abundance of deuteronlike $n p$-SRC pairs in nucleus $A$ relative to the deuteron is accurate to about $20 \%$. This has significant implications for planned 


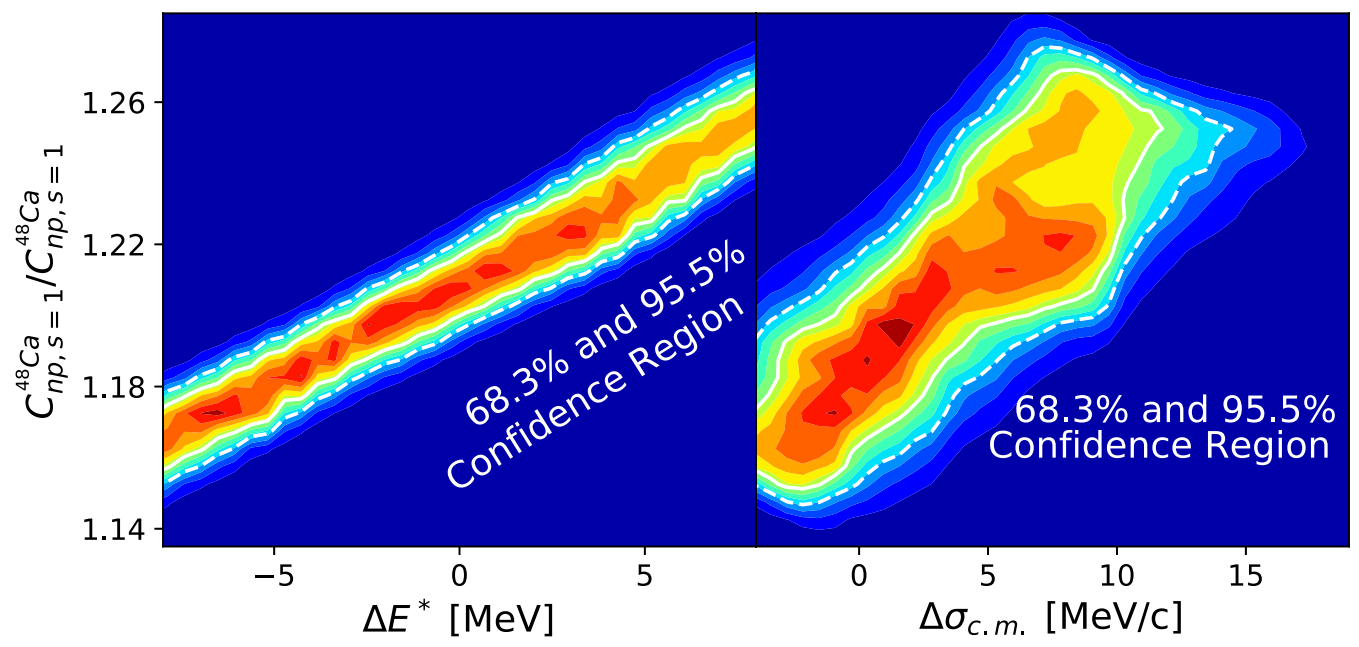

FIG. 4. Likelihood map for the correlation between the extracted ratio of spin-1 $p n$ contacts in ${ }^{48} \mathrm{Ca}$ over ${ }^{40} \mathrm{Ca}, C_{p n, s=1}^{48 \mathrm{Ca}} / C_{p n, s=1}^{40 \mathrm{Ca}}$, and $\Delta E^{*}=$ $E_{46 \mathrm{~K}}^{*}-E_{38 \mathrm{~K}}^{*}($ left $)$ and $\Delta \sigma_{\text {c.m. }}=\sigma_{\text {c.m. }}^{48 \mathrm{Ca}}-\sigma_{\text {c.m. }}^{40 \mathrm{Ca}}$ (right). The parameters likelihoods are determined by fitting the ${ }^{40} \mathrm{Ca}\left(e, e^{\prime}\right) /{ }^{48} \mathrm{Ca}\left(e, e^{\prime}\right) \mathrm{cross}-$ section ratio data of Ref. [39] with GCF calculations in the $x_{B}$ range of $1.5 \leqslant x_{B} \leqslant 1.9$. The calculation used the GCF light-cone formulations with the AV18 NN interaction [56]. The color scale represents the likelihood of the fit parameters given the data, with the white solid (dashed) contours indicating the $68.3 \%$ (95.5\%) confidence regions. See text for details.

precision measurements [47] of the nuclear mass and asymmetry dependence of $a_{2}$, especially for light nuclei. While the cross-section ratio $a_{2}$ can be measured precisely, supplemental $\left(e, e^{\prime} N\right)$ and $\left(e, e^{\prime} N N\right)$ measurements and detailed crosssection calculations are needed for its accurate interpretation.

We thank M. Strikman, M. Sargsian, W. Cosyn, J. Ryckebusch, and C. Weiss for insightful discussions. This work was supported by the U.S. Department of Energy,
Office of Science, Office of Nuclear Physics under Grants No. DE-FG02-94ER40818, No. DE-SC0020240, No. DEFG02-96ER-40960, No. DE-FG02-93ER40771, and No. DEAC05-06OR23177 under which Jefferson Science Associates operates the Thomas Jefferson National Accelerator Facility, the Israeli Science Foundation (Israel) under Grants No. 136/12 and No. 1334/16, the Pazy foundation, and the Clore Foundation.

R.W. and A.W.D. contributed equally to this work.
[1] O. Hen, G. A. Miller, E. Piasetzky, and L. B. Weinstein, Rev. Mod. Phys. 89, 045002 (2017).

[2] C. Ciofi degli Atti, Phys. Rep. 590, 1 (2015).

[3] E. Piasetzky, M. Sargsian, L. Frankfurt, M. Strikman, and J. W. Watson, Phys. Rev. Lett. 97, 162504 (2006).

[4] R. Subedi et al., Science 320, 1476 (2008).

[5] M. Alvioli, C. Ciofi degli Atti, and H. Morita, Phys. Rev. Lett. 100, 162503 (2008).

[6] O. Hen et al., Science 346, 614 (2014).

[7] I. Korover et al. (Jefferson Lab Hall A Collaboration), Phys. Rev. Lett. 113, 022501 (2014).

[8] R. Weiss, R. Cruz-Torres, N. Barnea, E. Piasetzky, and O. Hen, Phys. Lett. B 780, 211 (2018).

[9] M. Duer et al. (CLAS Collaboration), Phys. Rev. Lett. 122, 172502 (2019).

[10] I. Korover et al. (CLAS Collaboration), arXiv:2004.07304.

[11] S. Tan, Ann. Phys. (NY) 323, 2952 (2008).

[12] S. Tan, Ann. Phys. (NY) 323, 2971 (2008).

[13] S. Tan, Ann. Phys. (NY) 323, 2987 (2008).

[14] E. Braaten, in The BCS-BEC Crossover and the Unitary Fermi Gas, edited by W. Zwerger (Springer-Verlag, Berlin, 2012).

[15] R. Weiss, B. Bazak, and N. Barnea, Phys. Rev. Lett. 114, 012501 (2015).
[16] R. Weiss, B. Bazak, and N. Barnea, Phys. Rev. C 92, 054311 (2015)

[17] R. Cruz-Torres, A. Schmidt, G. A. Miller, L. B. Weinstein, N. Barnea, R. Weiss, E. Piasetzky, and O. Hen, Phys. Lett. B 785, 304 (2018).

[18] R. Cruz-Torres, D. Lonardoni, R. Weiss, N. Barnea, D. W. Higinbotham, E. Piasetzky, A. Schmidt, L. B. Weinstein, R. B. Wiringa, and O. Hen, Nat. Phys. 17, 306 (2021).

[19] R. Weiss, I. Korover, E. Piasetzky, O. Hen, and N. Barnea, Phys. Lett. B 791, 242 (2019).

[20] A. Schmidt et al. (CLAS Collaboration), Nature (London) 578, 540 (2020).

[21] O. Hen, B.-A. Li, W.-J. Guo, L. B. Weinstein, and E. Piasetzky, Phys. Rev. C 91, 025803 (2015).

[22] B.-J. Cai and B.-A. Li, Phys. Rev. C 93, 014619 (2016).

[23] B.-A. Li, B.-J. Cai, L.-W. Chen, and J. Xu, Prog. Part. Nucl. Phys. 99, 29 (2018).

[24] L. A. Souza, R. Negreiros, M. Dutra, D. P. Menezes, and O. Lourenço, arXiv:2004.10309.

[25] L. B. Weinstein, E. Piasetzky, D. W. Higinbotham, J. Gomez, O. Hen, and R. Shneor, Phys. Rev. Lett. 106, 052301 (2011).

[26] O. Hen, E. Piasetzky, and L. B. Weinstein, Phys. Rev. C 85, 047301 (2012). 
[27] O. Hen, D. W. Higinbotham, G. A. Miller, E. Piasetzky, and L. B. Weinstein, Int. J. Mod. Phys. E 22, 1330017 (2013).

[28] J.-W. Chen, W. Detmold, J. E. Lynn, and A. Schwenk, Phys. Rev. Lett. 119, 262502 (2017).

[29] C. Ciofi degli Atti, L. L. Frankfurt, L. P. Kaptari, and M. I. Strikman, Phys. Rev. C 76, 055206 (2007).

[30] B. Schmookler et al. (CLAS Collaboration), Nature (London) 566, 354 (2019).

[31] J. Arrington and N. Fomin, Phys. Rev. Lett. 123, 042501 (2019).

[32] O. Hen, F. Hauenstein, D. W. Higinbotham, G. A. Miller, E. Piasetzky, A. Schmidt, E. P. Segarra, M. Strikman, and L. B. Weinstein, arXiv:1905.02172.

[33] O. Hen, A. Accardi, W. Melnitchouk, and E. Piasetzky, Phys. Rev. D 84, 117501 (2011).

[34] E. P. Segarra, A. Schmidt, T. Kutz, D. W. Higinbotham, E. Piasetzky, M. Strikman, L. B. Weinstein, and O. Hen, Phys. Rev. Lett. 124, 092002 (2020).

[35] L. L. Frankfurt, M. I. Strikman, D. B. Day, and M. Sargsyan, Phys. Rev. C 48, 2451 (1993).

[36] K. Egiyan et al. (CLAS Collaboration), Phys. Rev. C 68, 014313 (2003).

[37] K. Egiyan et al. (CLAS Collaboration), Phys. Rev. Lett. 96, 082501 (2006).

[38] N. Fomin et al., Phys. Rev. Lett. 108, 092502 (2012).

[39] D. Nguyen et al. (Jefferson Lab Hall A Collaboration), Phys. Rev. C 102, 064004 (2020).

[40] E. O. Cohen et al. (CLAS Collaboration), Phys. Rev. Lett. 121, 092501 (2018).

[41] L. L. Frankfurt and M. I. Strikman, Phys. Rep. 76, 215 (1981).

[42] L. Frankfurt and M. Strikman, Phys. Rep. 160, 235 (1988).

[43] C. Ciofi degli Atti and S. Simula, Phys. Lett. B 325, 276 (1994).

[44] C. Ciofi degli Atti and S. Simula, Phys. Rev. C 53, 1689 (1996).

[45] O. Benhar, A. Fabrocini, S. Fantoni, and I. Sick, Phys. Lett. B 343, 47 (1995).

[46] L. Frankfurt, M. Sargsian, and M. Strikman, Int. J. Mod. Phys. A 23, 2991 (2008).

[47] N. Fomin, D. Higinbotham, M. Sargsian, and P. Solvignon, Annu. Rev. Nucl. Part. Sci. 67, 129 (2017).

[48] R. Schiavilla, R. B. Wiringa, S. C. Pieper, and J. Carlson, Phys. Rev. Lett. 98, 132501 (2007).

[49] H. Feldmeier, W. Horiuchi, T. Neff, and Y. Suzuki, Phys. Rev. C 84, 054003 (2011).

[50] T. Neff, H. Feldmeier, and W. Horiuchi, Phys. Rev. C 92, 024003 (2015).
[51] J. Carlson, S. Gandolfi, and A. Gezerlis, Prog. Theor. Exp. Phys. 2012, 01A209 (2012).

[52] R. B. Wiringa, R. Schiavilla, S. C. Pieper, and J. Carlson, Phys. Rev. C 89, 024305 (2014).

[53] J. Lynn, D. Lonardoni, J. Carlson, J. Chen, W. Detmold, S. Gandolfi, and A. Schwenk, J. Phys. G 47, 045109 (2020).

[54] J. Pybus, I. Korover, R. Weiss, A. Schmidt, N. Barnea, D. Higinbotham, E. Piasetzky, M. Strikman, L. Weinstein, and O. Hen, Phys. Lett. B 805, 135429 (2020).

[55] C. Colle, W. Cosyn, J. Ryckebusch, and M. Vanhalst, Phys. Rev. C 89, 024603 (2014).

[56] R. B. Wiringa, V. G. J. Stoks, and R. Schiavilla, Phys. Rev. C 51, 38 (1995).

[57] J. Ryckebusch, W. Cosyn, T. Vieijra, and C. Casert, Phys. Rev. C 100, 054620 (2019).

[58] R. B. Wiringa and S. C. Pieper, Phys. Rev. Lett. 89, 182501 (2002).

[59] M. Piarulli, L. Girlanda, R. Schiavilla, A. Kievsky, A. Lovato, L. E. Marcucci, S. C. Pieper, M. Viviani, and R. B. Wiringa, Phys. Rev. C 94, 054007 (2016).

[60] M. Piarulli et al., Phys. Rev. Lett. 120, 052503 (2018).

[61] A. Baroni, R. Schiavilla, L. E. Marcucci, L. Girlanda, A. Kievsky, A. Lovato, S. Pastore, M. Piarulli, S. C. Pieper, M. Viviani, and R. B. Wiringa, Phys. Rev. C 98, 044003 (2018).

[62] A. Gezerlis, I. Tews, E. Epelbaum, M. Freunek, S. Gandolfi, K. Hebeler, A. Nogga, and A. Schwenk, Phys. Rev. C 90, 054323 (2014).

[63] J. E. Lynn, I. Tews, J. Carlson, S. Gandolfi, A. Gezerlis, K. E. Schmidt, and A. Schwenk, Phys. Rev. Lett. 116, 062501 (2016).

[64] D. Lonardoni, S. Gandolfi, J. E. Lynn, C. Petrie, J. Carlson, K. E. Schmidt, and A. Schwenk, Phys. Rev. C 97, 044318 (2018).

[65] See Supplemental Material at http://link.aps.org/supplemental/ 10.1103/PhysRevC.103.L031301 for additional analysis details and results for additional nuclei and interactions.

[66] M. Vanhalst, J. Ryckebusch, and W. Cosyn, Phys. Rev. C 86, 044619 (2012).

[67] J. Arrington, A. Daniel, D. B. Day, N. Fomin, D. Gaskell, and P. Solvignon, Phys. Rev. C 86, 065204 (2012).

[68] M. M. Sargsian, D. B. Day, L. L. Frankfurt, and M. I. Strikman, Phys. Rev. C 100, 044320 (2019).

[69] M. M. Sargsian, Phys. Rev. C 89, 034305 (2014).

[70] M. McGauley and M. M. Sargsian, arXiv:1102.3973.

[71] S. N. More, S. K. Bogner, and R. J. Furnstahl, Phys. Rev. C 96, 054004 (2017). 